\title{
A Ligand-Induced Switch in the Periplasmic Domain of Sensor Histidine Kinase CitA
}

\author{
Madhumati Sevvana ${ }^{1}$, Vinesh Vijayan ${ }^{2}$, Markus Zweckstetter ${ }^{2}$, \\ Stefan Reinelt ${ }^{3}$, Dean R. Madden ${ }^{3}$, Regine Herbst-Irmer ${ }^{1}$, \\ George M. Sheldrick ${ }^{1}$, Michael Bott ${ }^{4}$, Christian Griesinger ${ }^{2}$ \\ and Stefan Becker ${ }^{2 *}$
}

\author{
${ }^{1}$ Department of Structural \\ Chemistry, University of \\ Göttingen, Tammannstrasse 4, \\ 37077 Göttingen, Germany \\ ${ }^{2}$ Department of NMR-Based \\ Structural Biology, Max Planck \\ Institute for Biophysical \\ Chemistry, Am Faßberg 11, \\ 37077 Göttingen, Germany \\ ${ }^{3}$ Department of Biochemistry, \\ Dartmouth Medical School, \\ 7200 Vail Building, Hanover, \\ NH 03755-3844, USA \\ ${ }^{4}$ Institute of Biotechnology 1, \\ Research Centre Jülich, \\ 52425 Jülich, Germany
}

Received 21 October 2007; received in revised form 8 January 2008; accepted 10 January 2008 Available online 16 January 2008

Edited by R. Huber

\begin{abstract}
Sensor histidine kinases of two-component signal-transduction systems are essential for bacteria to adapt to variable environmental conditions. However, despite their prevalence, it is not well understood how extracellular signals such as ligand binding regulate the activity of these sensor kinases. CitA is the sensor histidine kinase in Klebsiella pneumoniae that regulates the transport and anaerobic metabolism of citrate in response to its extracellular concentration. We report here the X-ray structures of the periplasmic sensor domain of CitA in the citrate-free and citrate-bound states. A comparison of the two structures shows that ligand binding causes a considerable contraction of the sensor domain. This contraction may represent the molecular switch that activates transmembrane signaling in the receptor.
\end{abstract}

(C) 2008 Elsevier Ltd. All rights reserved.
*Corresponding author. E-mail address:

sabe@nmr.mpibpc.mpg.de.

Present addresses: M. Sevvana, Lehrstuhl für Biotechnik, Universität Erlangen-Nürnberg, IZMP, Henkestr. 91, 91052 Erlangen, Germany; S. Reinelt, Basilea Pharmaceutica AG, Grenzacherstr. 487, 4005 Basel, Switzerland.

Abbreviations used: PAS, Per-Arnt-Sim; MAD, multiwavelength anomalous diffraction; r.m.s.d., root mean square deviation; TCS, two-component system; HK, histidine kinase; RR, response regulator; TM2, second transmembrane $\alpha$ helix; HSQC, heteronuclear single quantum coherence; NOE, nuclear Overhauser enhancement; RDCs, residual dipolar couplings; CNS, Crystallography \& NMR System.

\section{Introduction}

Bacteria respond efficiently to a large number of changes in their environment with the help of signaltransduction systems. For a plethora of signals, this adaptability is mediated by two-component regulatory systems. ${ }^{1}$ Generally, these two-component systems (TCSs) consist of a sensor histidine kinase (HK) and a response regulator (RR) protein. Class I HKs ${ }^{2}$ are usually homodimeric membrane proteins. Each monomer contains a short $\mathrm{N}$-terminal cytoplasmic domain followed by a transmembrane $\alpha$ helix and a periplasmic sensory domain that is connected via a second transmembrane $\alpha$ helix (TM2) to the cytoplasmic C-terminal HK domain. ${ }^{3}$ The HK domain is 
itself composed of a catalytic domain and a dimerization domain, and extracellular signals trigger transphosphorylation of a specific His residue on the dimerization domain. The phosphoryl group is then transferred to an Asp residue on the N-terminal receiver domain of $R R$, resulting in a cellular response via its $\mathrm{C}$-terminal effector domain. The sensory domain shows extremely low sequence similarity among different two-component sensory systems. However, in all structures solved so far, either cytoplasmic or periplasmic, these domains belong to the $\alpha / \beta$ protein class and most of them belong specifically to the Per-Arnt-Sim (PAS) domain superfamily, ${ }^{4-7}$ based on three-dimensional fold comparisons.

The sensor HK CitA of Klebsiella pneumoniae forms a TCS with the RR CitB. This system is essential for the induction of citrate fermentation genes under anoxic conditions in the presence of environmental citrate. ${ }^{8}$ These genes, which are organized on a gene cluster, 8,9 are tightly regulated, as they have immediate influence on central metabolic pathways. Therefore, although this TCS is nonessential, erroneous signaling by $\mathrm{Cit} A / \mathrm{CitB}$ could inhibit growth of K. pneumoniae. ${ }^{9}$ Thus, CitA, like sensor HKs of other pathogenic bacteria, is a potential therapeutic target, in particular because no TCS has as yet been identified in mammalian genomes.

Ligand binding to the periplasmic sensory domain of sensor HKs constitutes the trigger for subsequent transmembrane signaling events, leading to quaternary structural changes within the dimer and ultimately to $\mathrm{HK}$ autophosphorylation in trans. Understanding ligand binding on the atomic level is therefore a key requirement to gain insight into the nature of the associated conformational changes and the mechanism of signal transduction to the cytoplasmic kinase domain. Citrate binds to the recombinant sensory domain of CitA (CitAP) with high specificity. ${ }^{10,11}$ The structures of both the ligand-free and ligand-bound states are required to obtain insight into the coupling of ligand-induced conformational changes with the signal-transduction mechanism for a specific sensor domain. However, for CitA and for most other sensory HKs studied to date, the structure of only one binding state of their sensory domain is available, ${ }^{6,7,12}$ preventing solid mechanistic interpretations. Recently, the signal-transduction mechanism of the sensor kinase complex LuxPQ has been elucidated. ${ }^{13,14}$ The LuxQ HK is regulated by the periplasmic protein LuxP, which undergoes a major conformational change upon binding the ligand AI-2. As a result, the dimeric $(\text { LuxPQ })_{2}$ switches from a symmetric state to an asymmetric dimer, where one LuxP molecule interacts with both LuxQ periplasmic domains, while the second LuxP molecule contacts only one of the LuxQ domains.

Citrate sensing by the CitA periplasmic domain does not require complex formation with another protein. Therefore, signal transduction in CitA must employ a mechanism unrelated to the ligand-induced asymmetry mechanism of LuxPQ. The avail- able citrate-bound CitAP structure (Protein Data Bank code 1P0Z) shows the typical PAS domain fold and a dimerization mode with a highly suggestive clustering of the membrane attachment points ${ }^{6}$ that fits structurally to the dimeric model of sensory HKs. In addition to citrate, several metal centers have been modeled in this structure: a putative $\mathrm{Na}^{+}$in the neighborhood of the citrate-binding pocket, a $\mathrm{MoO}_{3}$

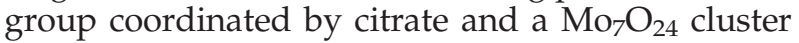
near the $\mathrm{N}$ - and C-termini. Although citrate frequently acts as a metal chelator in biological systems, a role for molybdenum in citrate transport and metabolism has not been described; thus, the presence of $\mathrm{MoO}_{3}$ in the binding site may represent a nonphysiological interaction. Therefore, we aimed to not only solve the structure of citrate-free CitAP but also obtain a citrate-bound structure in the absence of molybdenum. In this article, we describe the structures of citrate-bound, molybdenum-free CitAP and citrate-free CitAP. Comparison of these two structures clearly reveals a fundamentally intramolecular conformational trigger for transmembrane signaling across the membrane in response to citrate binding.

\section{Results and Discussion}

\section{Structure of citrate-bound CitAP}

A systematic search for molybdate-free crystallization conditions of citrate-bound CitAP resulted in crystals that diffracted to about $1.6 \AA$. The structure was solved by multiwavelength anomalous diffraction (MAD) phasing using data from a single Se-Met crystal. There are two monomers in the asymmetric unit [citrate-bound A (residues 4-132) and citratebound B (residues 6-129)] that form a dimer. Structurally (Figs. 1a and b), each monomer has a PAS domain fold. Citrate is bound by hydrogen bonding to the side chains of Thr58, Arg107, Lys109 and Ser124 from the $\beta$-sheet, to the side chain of Ser101 and the backbone carbonyl of Leu102 on the minor loop (residues 96-106) and to the side chains of Arg66 and His69 from the major loop (residues 63-92) of the structure (Fig. 1c). The major loop and the minor loop close the binding pocket, forming a tight lid over the bound citrate (Figs. 1a and c). The N-terminal region of CitAP has a helical conformation from residue 7 ; in the C-terminal region, there is a $3_{10}$ helix from residues 129 to 131 (monomer A). Several lattice contacts are made by the N-terminal residues, and the conformation of both termini may be altered by attachment to the transmembrane domains, so caution is advisable in interpreting the finer structural details of this region.

A superposition of citrate-bound A with monomer $\mathrm{G}$ in 1P0Z (Fig. 1d) shows that they superimpose quite well [root mean square deviation (r.m.s.d.) of all residues including side chains $=1.78 \AA]$. Also, bound citrate and $\mathrm{Na}^{+}$in both structures superimpose nearly perfectly, and their binding modes are very similar (Figs. $1 \mathrm{c}$ and d). Due to the $\mathrm{Mo}_{7} \mathrm{O}_{24}$ 
(a)

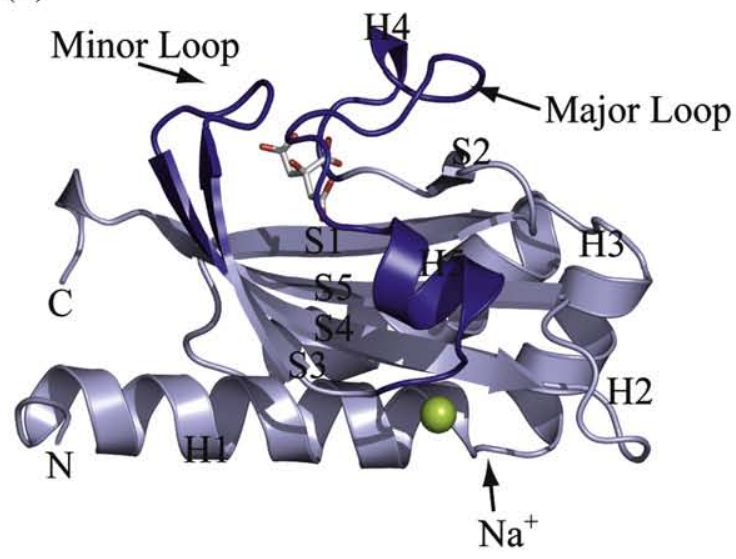

(c)

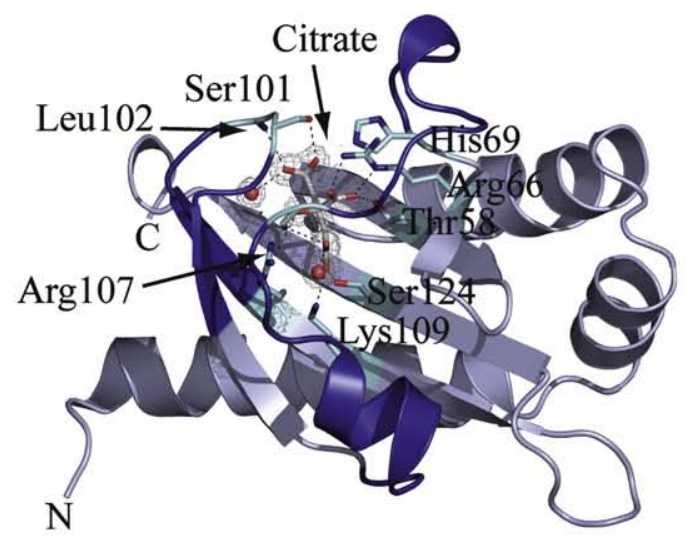

(e)

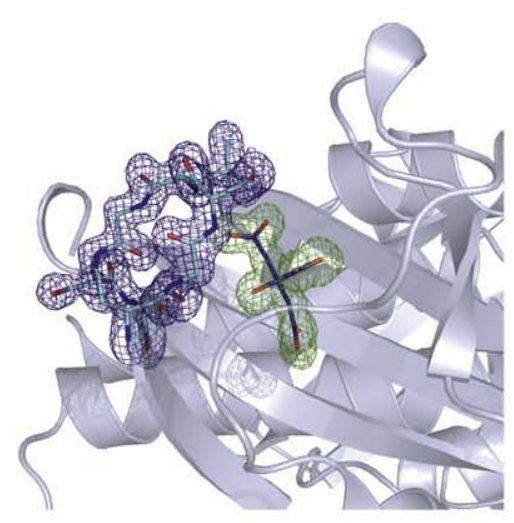

(b)

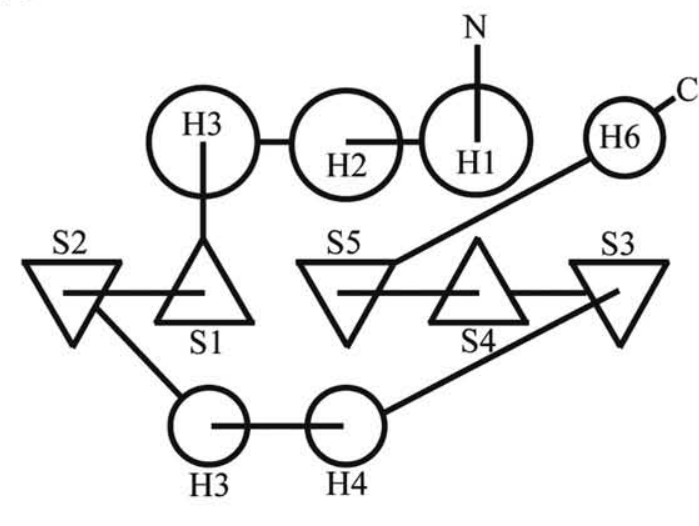

(d)

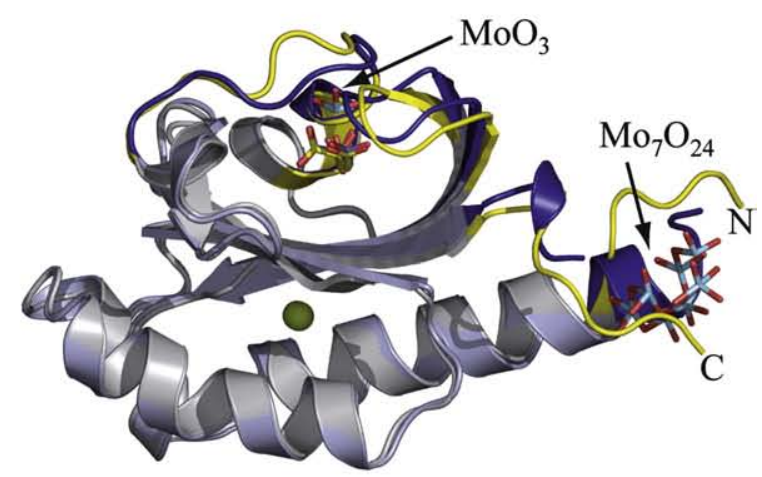

(f)

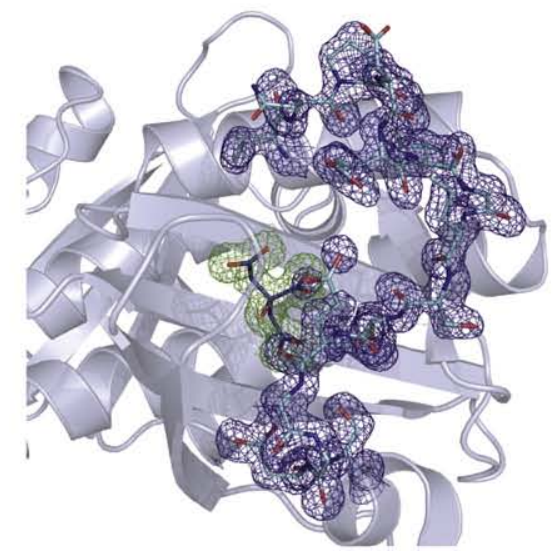

Fig. 1. Citrate-bound structure of CitAP. (a) The secondary structural elements, major loop residues and minor loop residues are highlighted in blue, bound $\mathrm{Na}^{+}$is highlighted in green and the bound citrate is in stick representation. (b) Topology diagram generated by TOPS ${ }^{15}$ of citrate-bound A. Circles symbolize $\alpha$ helices, and triangles are $\beta$-strands running roughly perpendicular through the plane of the page. Connecting segments penetrating into the symbols pass above the plane, while those stopping at the boundary of the symbol pass below it. H6 is the C-terminal 310 helix that is absent in citrate-bound B. (c) Citrate-binding site and residues involved in binding citrate. (d) Superposition of 1P0Z (differences are shown in dark blue) on our citrate-bound structure (differences are shown in yellow). Citrate is shown in stick representation (yellow: new structure; blue: $1 \mathrm{POZ}$ ). $\mathrm{MoO}_{3}$ near the active site and bound $\mathrm{Mo}_{7} \mathrm{O}_{24}$ near the $\mathrm{N}$ - and $\mathrm{C}$-termini of $1 \mathrm{POZ}$ are shown in stick representation. (e and f) Experimental maps contoured at $1 \sigma(\mathrm{e})$ along the minor loop residues (blue) and citrate (green) in the citrate-binding site and (f) along the major loop residues (blue) and citrate (green) in the citrate-bound form. 
cluster in $1 \mathrm{P} 0 \mathrm{Z}$, the $\mathrm{N}$ - and C-termini of both structures deviate substantially (Fig. 1d). The $\mathrm{MoO}_{3}$ next to citrate in $1 \mathrm{P} 0 \mathrm{Z}$ causes a more open conformation of the major and minor loops compared with the $\mathrm{MoO}_{3}$-free structure reported here.

To investigate the conformational dynamics of the CitAP domain in solution, we also performed NMR spectroscopy. Resonances in two-dimensional ${ }^{1} \mathrm{H} /{ }^{15} \mathrm{~N}$ heteronuclear single quantum coherence (HSQC) spectra were well dispersed, indicating that citrate-bound CitAP is well folded in solution (Fig. 2a) and is predominantly monomeric, in line with gel filtration data. ${ }^{6}$ Of 129 possible spin systems, 101 could be identified in the spectra, of which 100 were assigned using triple-resonance spectra. Undetectable residues were mainly located in the N-terminal dimerization helix, the loop connecting helix $\mathrm{H} 3$ and the first $\beta$-strand S1, and in the minor loop (residues 96-106), connecting $\beta$-strands S3 and $S 4 . C^{\alpha} / C^{\prime}$ secondary chemical shifts were in agreement with the secondary structure observed for citrate-bound CitAP in the crystalline environment (Fig. 2b). Secondary chemical shifts for residues 129-131 were slightly positive, suggesting the formation of a transient $\alpha$-helical structure in solution, consistent with the crystal structure. For most of the backbone of citrate-bound CitAP, there was only limited motion on the pico-to-nanosecond time scale as indicated by heteronuclear ${ }^{1} \mathrm{H} /{ }^{15} \mathrm{~N}$ nuclear Overhauser enhancement (NOE) values (Fig. 2c). This includes the major loop (residues 63-92), in which all assigned resonances had ${ }^{1} \mathrm{H} /{ }^{15} \mathrm{~N}$ NOE values above 0.7 .

\section{Dimerization}

The dimeric state of $\mathrm{HKs}$ is thought to be the competent form for signal transduction. ${ }^{3}$ In our citrate-bound CitAP crystal structure, a dimer is found in the asymmetric unit (Fig. 3a). Helix H1 forms a central parallel bundle involving residues 11-27, with helix H3 (residues 48-51) packed against them on either side (residues 21-25) (Fig. 3b). This dimerization mode results in a tightly packed dimer with an interface covering $2123 \AA^{2}$ of accessible surface area. The interface is not predominantly hydrophobic, pointing towards a dynamic character of the interaction between both CitAP domains. The GJ-type dimer in 1P0Z is far less tightly packed, with an interface of only $1415 \AA^{2}$. The interface of this dimer is strongly hydrophobic (Supplemental Figs. $1 \mathrm{a}$ and $\mathrm{b}$ ). Thus, despite high structural similarity between the core domains of the monomers, the dimerization modes in our new citrate-bound CitAP structure and in the GJ-type dimer from 1P0Z are very different in size and nature. Given the more extensive interface observed, our citrate-bound dimer is more likely to represent the physiological association.

\section{Structure of citrate-free CitAP and comparison with the citrate-bound structure}

The structure of citrate-free CitAP was determined to a resolution of $2.0 \AA$ from a merohedrally twinned crystal (Fig. 4a). There are two monomers in the asymmetric unit. We could not identify an ordered $\mathrm{Na}^{+}$ion. In monomer A, residues 4-130 were traced, except for the major loop (residues 68-89). In monomer B, residues 4-128, except for most of the major loop residues (69-88), were traced. Both monomers are structurally very similar (r.m.s.d. $=0.342 \AA$ ).

The absence of clear electron density for the major loop residues suggests that this region has high mobility in the absence of citrate. Support for this view also comes from NMR data of citrate-free CitAP. Strong line broadening was observed, and only 80 of the 129 expected backbone ${ }^{1} \mathrm{H} /{ }^{15} \mathrm{~N}$ signals

(b)

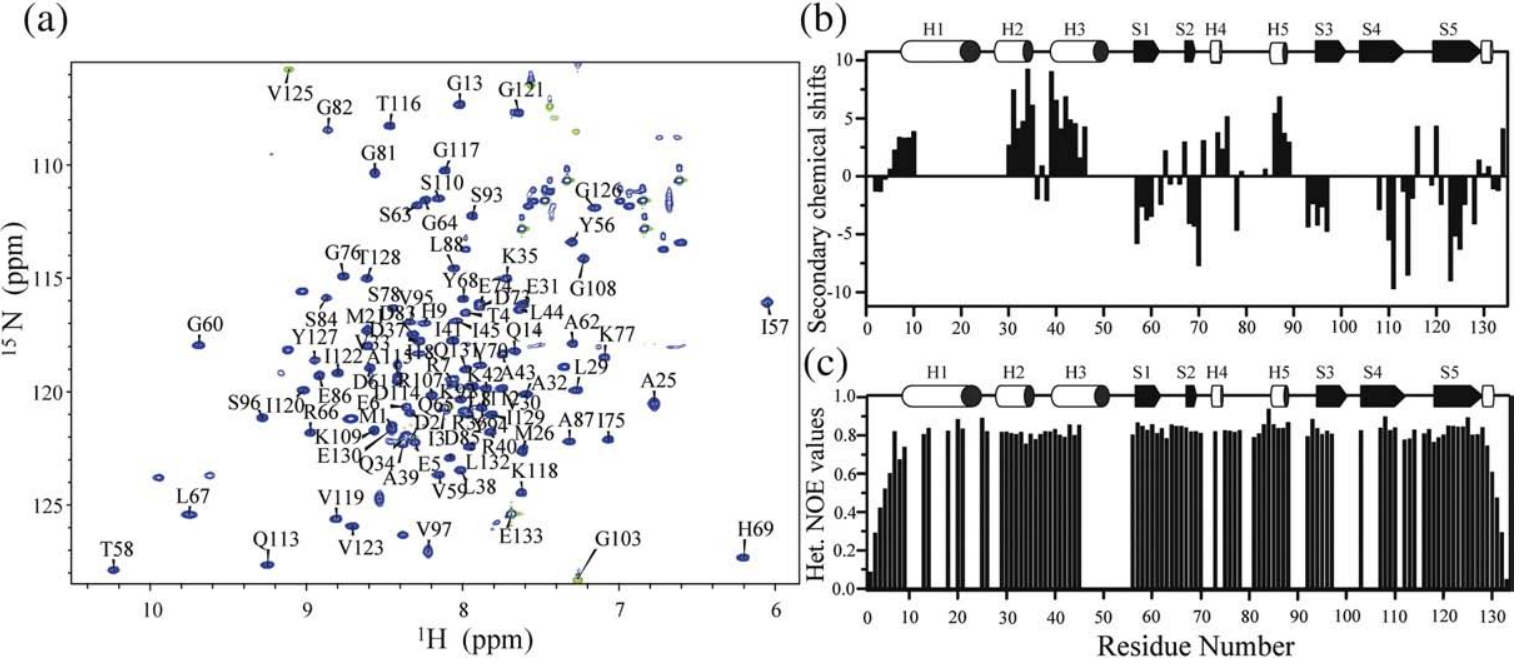

Fig. 2. Structure and dynamics of citrate-bound CitAP in solution as monitored by multidimensional NMR spectroscopy. (a) Two-dimensional ${ }^{1} \mathrm{H} /{ }^{15} \mathrm{~N}$ HSQC spectrum. Resonance assignments are indicated with residue numbers. (b) $C^{\alpha} / C^{\prime}$ secondary chemical shifts (secondary chemical shift=difference between experimental chemical shifts and random coil values). Regular secondary structure elements as observed in the crystal structure are indicated. (c) Heteronuclear ${ }^{1} \mathrm{H}-{ }^{15} \mathrm{~N}$ NOE values. Values below 0.7 are found for residues with increased dynamics. 


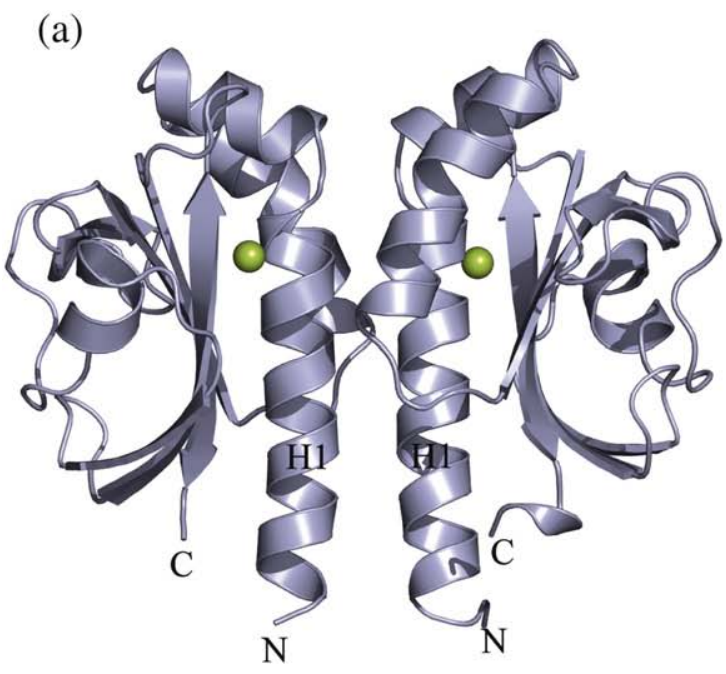

(b)

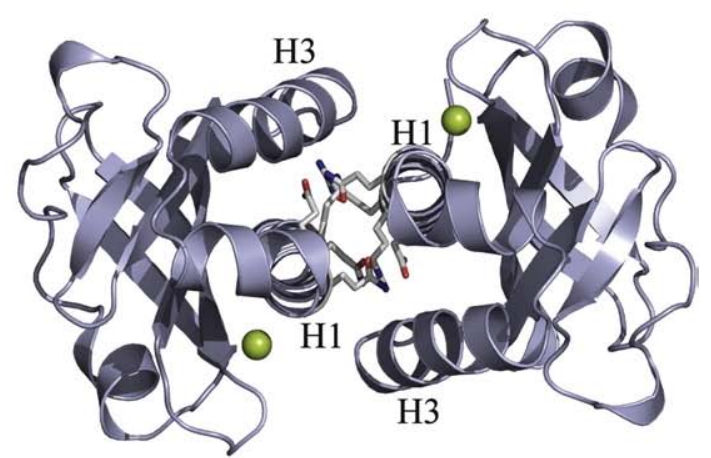

Fig. 3. Dimerization mode. (a) Dimer in the asymmetric unit of our citrate-bound structure showing bound $\mathrm{Na}^{+}$(green spheres). (b) Top view of the dimer in the asymmetric unit of our citrate-bound structure showing the interface residues.

were observed (Fig. 4b). In particular, NMR signals for residues in the major loop and in strands S1, S2 and S3 were broadened beyond detection by chemical exchange intermediate on the NMR time scale (Fig. 4c). For residues at both ends of the major loop (that were still observable in the citrate-free state), heteronuclear ${ }^{1} \mathrm{H} /{ }^{15} \mathrm{~N}$ NOE values indicated that pico-to-nanosecond dynamics were also increased (Fig. 4e). These data suggest that the major loop residues of citrate-free CitAP exchange between multiple conformations in solution. Notably, at the C-terminus, residues 129-131 also show strong changes in ${ }^{1} \mathrm{H} /{ }^{15} \mathrm{~N}$ chemical shifts (Fig. $4 \mathrm{c}$ ), have a lower propensity to form an $\alpha$-helical structure (Fig. 4d) and are more flexible in the citrate-free state (Fig. 4e).

The core structure of citrate-free CitAP is similar to our citrate-bound structure (Figs. 5a-c). Citrate-free monomer $\mathrm{A}$ has an r.m.s.d. (main chain $\mathrm{C}^{\alpha}$ atoms only) of $1.06 \AA$ in the central five-stranded core $\beta-$ sheet region when superposed on citrate-bound monomer A. Similarly, the positions and side chain orientations of the citrate-binding residues located in the core domain (Thr58, Ser124, Arg107 and Lys109; Fig. 5c) hardly change between citrate-bound CitAP and citrate-free CitAP. The rotamers of Thr58 and Ser124 are the same. Arg66 and His69, which both interact with citrate in the ligand-bound structure, cannot be localized in the ligand-free structure because they form part of the major loop that appears disordered in the absence of this interaction.

Furthermore, the r.m.s.d. of main chain atoms is significantly higher in the minor loop region $(4.76 \AA)$ than in the core (Figs. 5a and b). In part, this is because the central five-stranded antiparallel $\beta$-sheet is significantly less bent in the citrate-free state than it is in the citrate-bound state (Fig. 5a). In addition, the minor loop itself undergoes a structural rearrangement. In the citrate-bound state, it forms a typical type I $\beta$-turn from Gly100 to Gly103. Residues Gly103, Ser104 and Ser105 also form a $\gamma$-turn with a weak hydrogen bond between the main chain carbonyl of Gly103 and the main chain amide of Ser105
(Fig. 5d). In the ligand-free structure, the weaker bend of the $\beta$-sheet results in the loss of this $\gamma$-turn and the type I $\beta$-turn in the minor loop rearranges into a type II $\beta$-turn (Fig. 5e). This is remarkable because the position of Leu102 is nearly always occupied by a Gly residue in this type of $\beta$-turn. ${ }^{16}$ In view of the involvement of the Leu102 backbone amide in citrate binding (Fig. 5c), the switch between type II $\beta$-turn and type I $\beta$-turn is probably of functional relevance. The side chain of Ser101 is also involved in citrate binding (Figs. $1 c$ and $5 c$ ), providing an additional stereochemical connection between ligand binding and conformational change. As a result of these changes, the $C^{\alpha}$ of Ser101 at the tip of the minor loop has moved by a total of $13.54 \AA$ away from its position in citrate-bound CitAP (Figs. $5 a$ and $\mathrm{c}$ ).

Changes in the minor loop are coupled to the C-terminus of the domain, which also exhibits a higher $C^{\alpha}$ r.m.s.d. than the core (1.93 versus $\left.1.06 \AA\right)$. As mentioned above, the widening of the $\gamma$-turn between Gly103 and Ser105 accompanies the transition from the strongly bent $\beta$-sheet of ligand-bound CitAP to the ligand-free form. In parallel, the peptide bond between Ile129 and Glu130 of $\beta$-strand S5 flips between ligand-bound CitAP and ligand-free CitAP (the dihedral angles $\phi$ and $\psi$ of Ile129 are $-57.1^{\circ}$ and $-40.1^{\circ}$ in the ligand-bound state and $-124.2^{\circ}$ and $99.5^{\circ}$ in the ligand-free state, respectively). Taken together, these structural changes allow the formation of an additional hydrogen bond between the Ser104 backbone amide and the Ile129 backbone carbonyl (Figs. 5d and e). The typical $\beta$-sheet-type backbone hydrogen-bonding pattern between strands S4 and S5 is thereby extended by one more hydrogen bond upon transition from the ligandbound form to the ligand-free form of CitAP.

NMR residual dipolar couplings (RDCs) are exquisitely sensitive to bond vector orientations ${ }^{17}$ and are therefore uniquely suited for characterizing bending of $\beta$-sheets and $\alpha$ helices. For identical structures in solution and in the crystal, one can therefore 
(a)

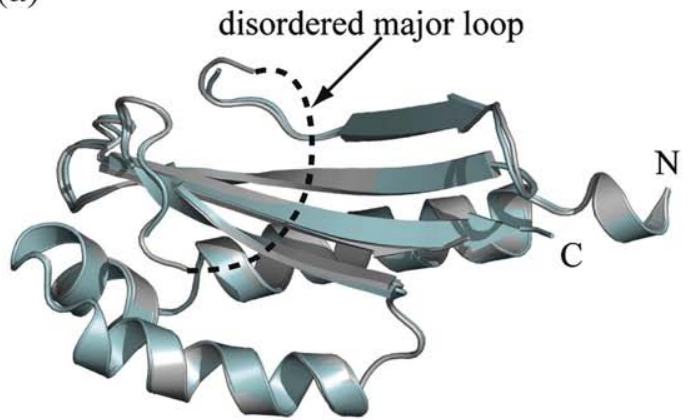

(b)

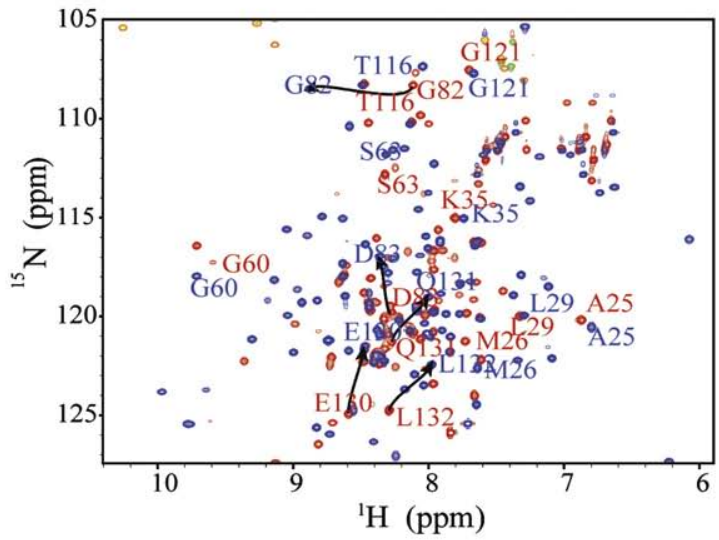

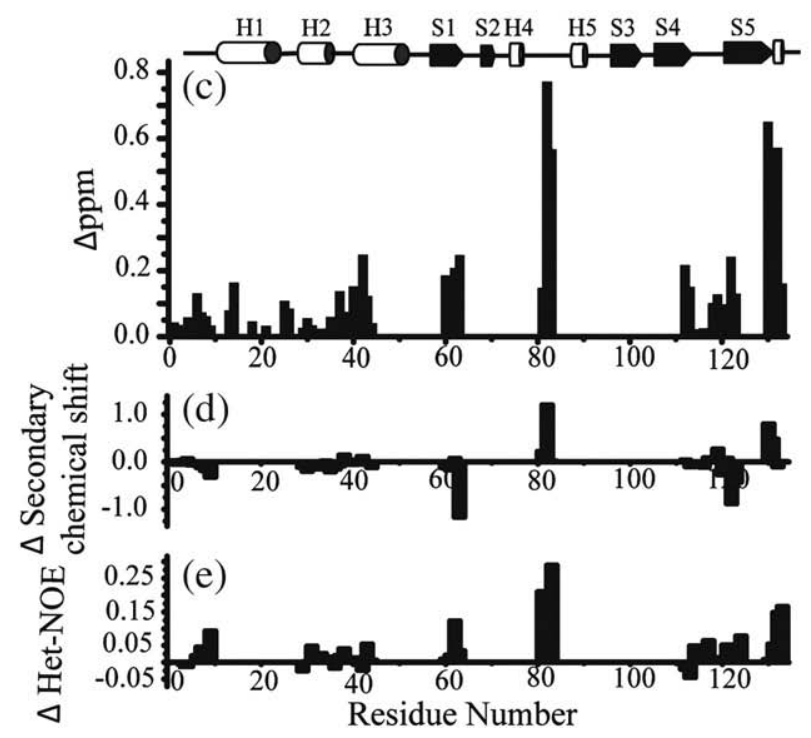

(f)

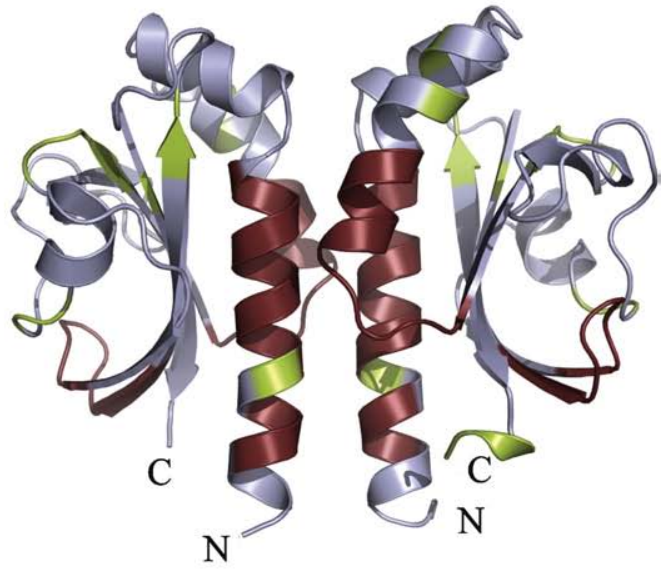

Fig. 4. Structure and dynamics of citrate-free CitAP. (a) Crystal structure of citrate-free CitAP with superposition of both monomers in the asymmetric unit. For one of the monomers, the missing major loop is indicated by a black broken line. (b-f) Comparison of the structure and dynamics of citrate-free CitAP and citrate-bound CitAP in solution. (b) Twodimensional ${ }^{1} \mathrm{H} /{ }^{15} \mathrm{~N}$ HSQC spectra of citrate-free CitAP (red) and citrate-bound CitAP (blue). Strongly shifting NMR resonances are highlighted. (c) Averaged ${ }^{1} \mathrm{H} /{ }^{15} \mathrm{~N}$ chemical shift differences. (d) Difference between heteronuclear ${ }^{1} \mathrm{H}-{ }^{15} \mathrm{~N}$ NOE values between the citrate-bound state and the citrate-free state. (e) Difference between $\mathrm{C}^{\alpha} / \mathrm{C}^{\prime}$ secondary chemical shifts in the citrate-bound state and those in the citrate-free state. (f) Ribbon representation of citrate-bound CitAP with residues that show strong chemical shift changes in green. Residues that were unassigned in citrate-free CitAP are shown in red.

expect an excellent fit of RDCs measured in solution to those parts of a given crystal structure for which experimental RDCs are available. It has also been observed that the quality of the fit of the RDCs decreases with decreasing quality of the structure. To this end, we measured and could observe onebond $\mathrm{NH}$ RDCs for 62 residues in citrate-bound CitAP. For citrate-free CitAP, RDCs could be reliably determined only for 32 residues due to the less complete assignment and the increased line broadening. The subset of residues for which RDCs could be observed in both citrate-free CitAP and citratebound CitAP comprised 26 residues (of the 32 observed for citrate-free CitAP). These 26 residues were mainly located in those parts of the structure that did not change strongly between the free and bound crystal structures and where the differences between these two structures were minimal. Only for citrate-bound CitAP were RDCs also observed in those regions that changed structure between free and bound states. The 62 RDCs observed for citratebound CitAP fit very well with RDCs backcalculated from the bound crystal structure, with a Pearson's correlation coefficient $(R)$ of 0.97 , but very badly $(R=0.69)$ to the free CitAP structure (Supplemental Fig. 2). ${ }^{18}$ This indicates that in solution, in the presence of citrate, the bound crystal structure prevails and the free crystal structure is, if at all, present only with a very minor population. Best fitting the 32 RDCs of citrate-free CitAP in solution to the crystal structures of citrate-free CitAP and citrate-bound CitAP resulted in correlation coefficients of 0.91 and 0.94 , respectively. These indicate that both crystal structures are compatible with the prevailing structure in solution for the region that is characterized by the common 26 RDCs (see above). 
(a)

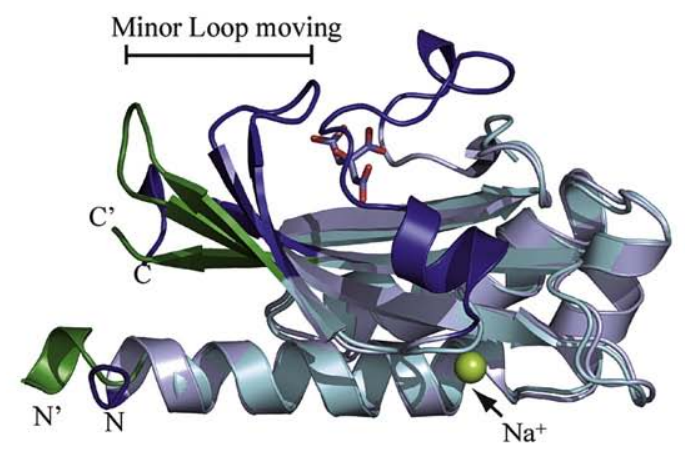

(b)

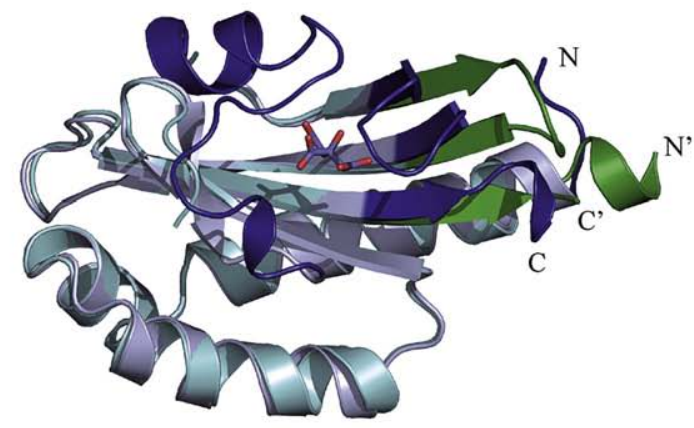

(c)

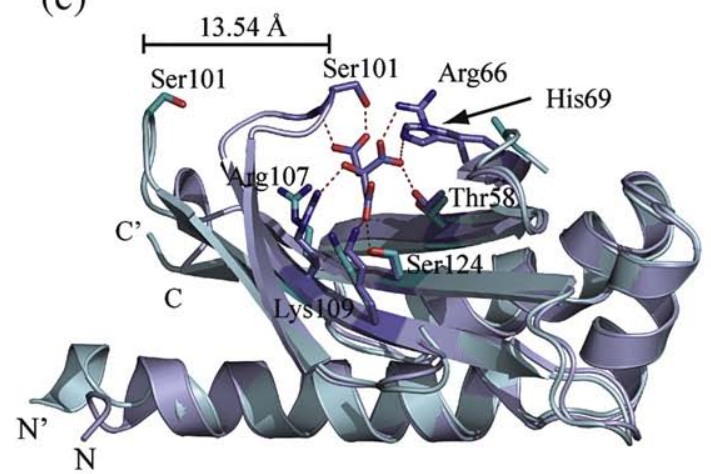

(d)

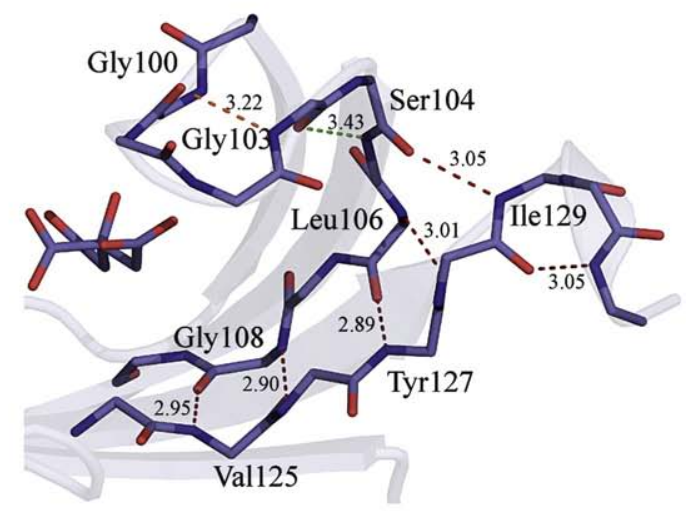

(e)

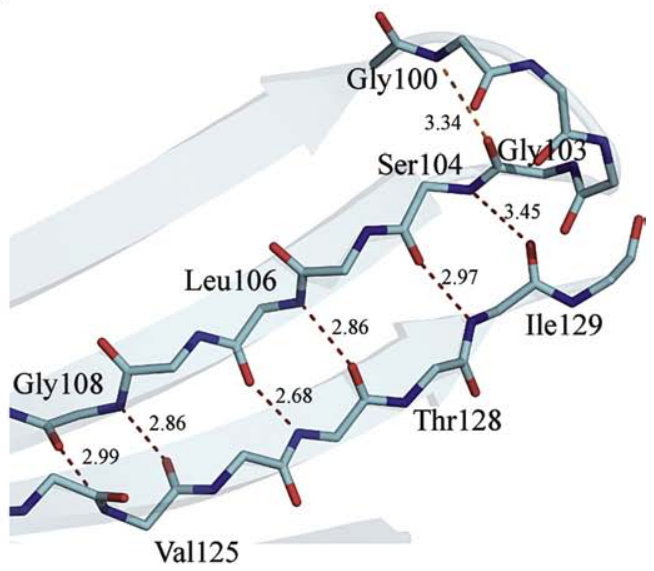

Fig. 5. Conformational differences between citrate-bound CitAP and citrate-free CitAP. (a) Side view of superposition of the citrate-bound structure (in light blue; differences highlighted in dark blue) on the citrate-free structure (monomer A) (in cyan; differences highlighted in green) showing the minor loop movement. (b) View from the opposite side of the superposition of the citrate-bound CitAP (differences highlighted in dark blue) on the citrate-free structure (differences highlighted in green) showing the conformational difference in the C-terminal region. (c) Side view similar to (a). The residues interacting with citrate as well as citrate are depicted as sticks (side chain carbon atoms of bound form in dark blue and side chain carbon atoms of free form in dark green). For clarity, the major loop is not shown for the bound form. ( $\mathrm{d}$ and e) Close-up views of the main chain hydrogen-bond pattern in the minor loop and between $\beta$-strands S4 and S5 (d) in the citrate-bound form of CitAP and (e) in the citrate-free form of CitAP. The backbone is shown in stick representation with a transparent secondary structure depiction in cartoon. The $\beta$-turn hydrogen bonds between $\beta$-strands S3 and S4 are depicted as orange broken lines, and the $\gamma$-turn hydrogen bond is depicted as a green broken line.

The slightly worse fit of the citrate-free crystal structure may well be due to its slightly lower quality compared with the citrate-bound crystal structure. This could be corroborated by fitting the 26 RDCs of citrate-bound CitAP in solution to the citrate-bound and citrate-free crystal structures. The correlation coefficients were 0.96 and 0.89 for citrate-bound CitAP and citrate-free CitAP, respectively. These suggest a lower quality of the citrate-free crystal structure, in agreement with the twinning of the crystals of citrate-free CitAP and the higher number of residues in the additionally and generously allowed regions of the Ramachandran plot (Table 1). In summary, NMR dipolar couplings demonstrate that the citrate-bound crystal structure prevails in solution. For the citrate-free structure in solution, 
Table 1. X-ray data collection, phasing and refinement statistics

\begin{tabular}{|c|c|c|c|c|}
\hline & \multicolumn{4}{|c|}{ CitA sensor domain } \\
\hline & \multicolumn{3}{|c|}{ Citrate bound $^{\mathrm{a}}$} & Citrate free \\
\hline & Peak & Inflection & $\begin{array}{l}\text { Low-energy } \\
\text { remote/Native }\end{array}$ & Native \\
\hline \multicolumn{5}{|l|}{ Data collection statistics } \\
\hline Wavelength $(\AA)$ & 0.97889 & 0.97920 & 1.0000 & 1.0000 \\
\hline X-ray source & \multicolumn{4}{|c|}{ BW6 (Max Planck Beamline DORIS III) } \\
\hline Detector & \multicolumn{4}{|c|}{ MarCCD } \\
\hline Space group & & $P 2{ }_{1} 2_{1} 2_{1}$ & & $I 4$ \\
\hline Unit cell parameters & \multicolumn{3}{|c|}{$a=53.21 \AA, b=54.79 \AA, c=116.33 \AA, \alpha=\beta=\gamma=90^{\circ}$} & $a=b=64.16 \AA, c=144.746 \AA, \alpha=\beta=\gamma=90^{\circ}$ \\
\hline Resolution $(\AA)^{\mathrm{b}}$ & $2.28(2.28-2.37)$ & $2.28(2.28-2.37)$ & $1.60(1.60-1.70)$ & $2.0(2.0-2.10)$ \\
\hline No. of reflections (unique) & 28,834 & 29,969 & 44,151 & 19,151 \\
\hline Redundancy ${ }^{\mathrm{b}}$ & $3.56(3.19)$ & $3.56(3.24)$ & $4.71(4.68)$ & $5.49(1.60)$ \\
\hline Completeness $(\%)^{\mathrm{b}}$ & $96.5(88.8)$ & $96.4(90.1)$ & $96.5(96.8)$ & $97.0(84.1)$ \\
\hline Mean $I /(\sigma I)^{\mathrm{b}}$ & $25.25(16.51)$ & $26.84(16.71)$ & $15.01(2.77)$ & $16.70(2.70)$ \\
\hline$R_{\text {int }}(\%)^{b, c}$ & $3.42(6.24)$ & $3.22(6.17)$ & $4.83(44.10)$ & $4.77(18.70)$ \\
\hline \multicolumn{5}{|l|}{ Refinement statistics } \\
\hline \multicolumn{5}{|l|}{ Final $R$-factor (\%) } \\
\hline Working set & & 19.29 & & 21.24 \\
\hline Working set+test set & & 19.42 & & - \\
\hline Final $R_{\text {free }}(\%)^{\mathrm{d}}$ & & 21.70 & & 27.83 \\
\hline \multicolumn{5}{|l|}{ r.m.s.d. } \\
\hline Bond lengths ( $)$ & & 0.025 & & 0.010 \\
\hline Bond angles $\left({ }^{\circ}\right)$ & & 1.498 & & 0.567 \\
\hline \multicolumn{5}{|l|}{ Mean $B$-value $\left(\AA^{2}\right)$} \\
\hline \multicolumn{5}{|l|}{ Monomer A } \\
\hline Main chain atoms & & 18.2 & & 22.2 \\
\hline Side chain atoms & & 21.1 & & 23.1 \\
\hline \multicolumn{5}{|l|}{ Monomer B } \\
\hline Main chain atoms & & 19.0 & & 22.7 \\
\hline Side chain atoms & & 21.8 & & 23.5 \\
\hline Solvent $(\%)$ & & 32.6 & & 28.3 \\
\hline No. of protein atoms & & 1877 & & 1496 \\
\hline No. of solvent atoms & & 259 & & 239 \\
\hline Twin fraction in Citrate free $(\%)^{\mathrm{e}}$ & & - & & 0.41 \\
\hline \multicolumn{5}{|c|}{ Ramachandran plot (\% of residues in) } \\
\hline Most favored regions & & 94 & & 84.8 \\
\hline Additionally allowed regions & & 6 & & 14.0 \\
\hline Generously allowed regions & & - & & 1.1 \\
\hline Disallowed regions & & - & & - \\
\hline $\begin{array}{l}\text { a For citrate-bound CitA, all da } \\
\text { b Values in parentheses are for } \\
\text { c } R_{\text {int }}=\sum \mid F_{\mathrm{o}}^{2}-F_{\mathrm{o}}^{2} \text { (mean) } \mid / \sum[F \\
\text { d Five percent of reflections in } \\
\text { e The twin fraction }(0.41) \text { was }\end{array}$ & $\begin{array}{l}\text { sets were meast } \\
\text { in shells were ta } \\
\text { iner than the pre }\end{array}$ & $\begin{array}{l}\text { as the } R_{\text {free }} \text { set. } \\
\text { cted value }(0.2) \mathrm{d}\end{array}$ & lionine derivative & a and non-crystallographic symmetry. \\
\hline
\end{tabular}

the structurally stable region (those 26 residues mentioned above) is identical with the crystal structure. For the other residues, the broadening of the resonances beyond detection gives support for the assumption that in solution the free structure exchanges among multiple conformations, which most likely include both the free and bound crystal structures.

It was shown previously that induction of the target genes by CitA/CitB requires not only citrate but also $\mathrm{Na}^{+}$. . This feature is physiologically meaningful as both citrate transport and metabolism are also strictly dependent on $\mathrm{Na}^{+} \cdot{ }^{19,20}$ Our structures are consistent with a functional connection between citrate and $\mathrm{Na}^{+}$binding to CitAP, as $\mathrm{Na}^{+}$was identified only in the citrate-bound CitAP structure. However, although strongly supported by bond valence calculations, ${ }^{21}$ the identity of the $\mathrm{Na}^{+}$modeled in this structure is not absolutely certain. Also, the fact that we could not identify an ordered $\mathrm{Na}^{+}$in the citrate-free form does not strictly exclude the possibility that $\mathrm{Na}^{+}$may be present but disordered. Therefore, further investigations will be necessary to obtain insight into the specific function of $\mathrm{Na}^{+}$for citrate binding by CitA.

\section{Implications for signal-transduction mechanism}

The major conformational rearrangement between the citrate-free and citrate-bound structures involves the flexing of the central $\beta$-sheet. In the citratebound molecules, the end of the $\beta$-sheet is pulled in towards the citrate-binding site, forming tight interactions with the activating ligand. This disrupts a strand-to-strand interaction between S4 and S5 and simultaneously pulls the C-terminus upwards, away from the membrane. Regardless of the exact geometry of the C-terminal attachment, these changes 
are likely to be communicated directly to the TM2 helix (Figs. 6a and b), causing an immediate transduction of ligand binding into a conformational change at the transmembrane junction.

The observed change occurs in close proximity to the bound ligand and involves multiple strands of the $\beta$-sheet. It is therefore unlikely to depend on the exact conformation of the C-terminus, which differs between the two models. In addition, the open minor loop and the extended S4-S5 structure seen in the citrate-free structure are formed in a region that experiences only weak, water-mediated lattice contacts, and the multiple main chain interactions stab- ilizing the extended structure suggest that it is likely to reflect the pre-signaling conformation of the receptor. In the citrate-bound molecules, the more closed structure of the $\beta$-sheet permits direct interaction of the minor loop with the ligand, providing a plausible stereochemical basis for the rearrangement. As a result, the conformational changes observed are likely to reflect distinct functional states associated with signal transduction.

Our attempts to express recombinant full-length CitA were not successful, preventing further biochemical and genetic investigations of the signaltransduction mechanism. However, the contraction

(a)

(b)
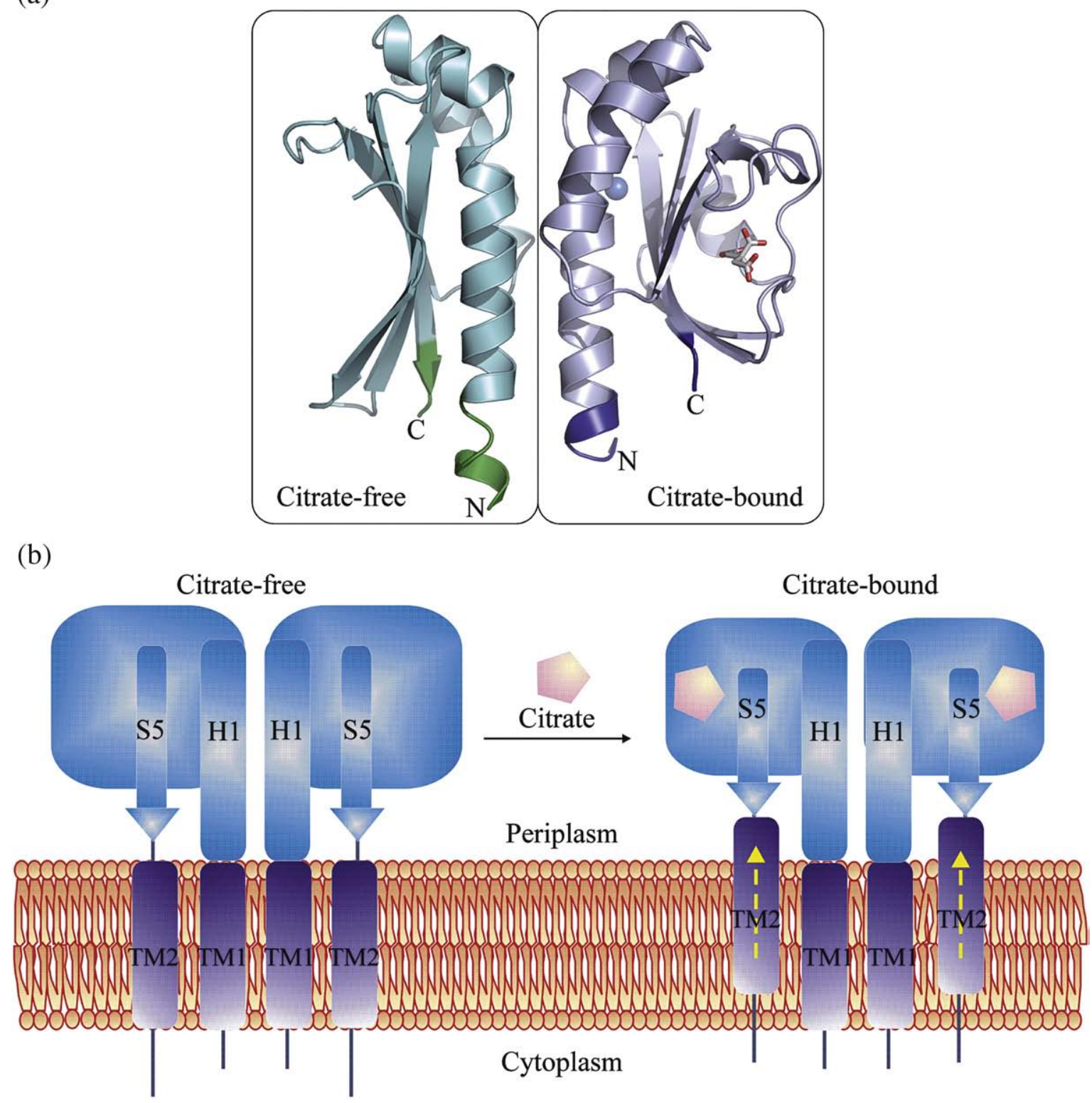

Fig. 6. Piston-type transmembrane signaling. (a) Comparison of the citrate-free A monomer (superposed on the citrate-bound B) with the citrate-bound A monomer. For clarity, the citrate-bound A monomer is only shown up to Gln130, the last ordered C-terminal residue in citrate-free A. (b) Hypothetical model of the ligand-induced switch in the periplasmic domain of CitA triggering a vectorial piston-type movement of TM2. The left part of the figure shows the citrate-free state, where the periplasmic PAS domain is in a relaxed state. Upon citrate binding (right part of the figure), the PAS domain contracts by the postulated flexing-type motion, thereby triggering a vectorial piston-type movement of TM2 and transduction of the signal to the cytoplasmic side. 
of the periplasmic domain of CitA upon ligand binding, as shown in this study, might indeed exert force onto the transmembrane helices. Four basic mechanisms for transducing a signal by transmembrane helices have been proposed: translation, piston-type displacement, rotation parallel to the membrane (pivot movement) and rotation perpendicular to the membrane. ${ }^{22-24}$ Our data presented here do not allow a definite decision as to which of these transduction mechanisms is used by CitA for signal transduction. Nevertheless, the flexing of the CitA sensory domain as shown in the structures presented here is in line with a contraction of the periplasmic domain likely to cause an axial displacement between the first transmembrane $\alpha$ helix and TM2, resulting in piston-type movement. Experimental evidence for the piston model was found for the transmembrane signaling of the aspartate receptor. $^{25}$ Such a vectorial movement (Fig. 6b) could be the trigger of kinase activity for the broad family of sensor kinases that do not employ an auxiliary signaling domain like that found in the LuxPQ system.

\section{Materials and Methods}

\section{Protein expression and purification}

The cDNA encoding fragment 45-176 of K. pneumoniae CitA (CitAP) was cloned into a modified pET16b vector containing a TEV cleavage site. This construct was transformed into the Escherichia coli strain BL21(DE3). The protein was expressed and purified as previously described. ${ }^{10}$ Se-Met-labeled CitAP was overexpressed in minimal media supplemented with selenomethionine according to the European Molecular Biology Laboratory protein expression group $\dagger .{ }^{15} \mathrm{~N}$-labeled CitAP and ${ }^{13} \mathrm{C}-$ labeled CitAP were expressed in M9 minimal media with ${ }^{15} \mathrm{NH}_{4} \mathrm{Cl}$ and ${ }^{13} \mathrm{C}_{6}$-D-glucose as the sole nitrogen and carbon sources, respectively.

\section{Crystallization and data collection}

Crystals of citrate-bound CitAP were obtained using the hanging drop vapor diffusion method by mixing $1.5 \mu \mathrm{l}$ of $20 \mathrm{mg} / \mathrm{ml}$ protein solution with $1.5 \mu \mathrm{l}$ of reservoir solution [0.1 M Hepes, $\mathrm{pH} 7.5,1.6 \mathrm{M}\left(\mathrm{NH}_{4}\right)_{2} \mathrm{SO}_{4}$ and $5 \mathrm{mM}$ sodium citrate]. For crystallization of the citrate-free CitAP, $1 \mu \mathrm{l}$ of $15-\mathrm{mg} / \mathrm{ml}$ protein solution was mixed with $1 \mu \mathrm{l}$ of reservoir solution (20 mM Hepes, pH 7.5, $0.63 \mathrm{M} \mathrm{NaH}_{2} \mathrm{PO}_{4}$ and $0.63 \mathrm{M} \mathrm{KH}_{2} \mathrm{PO}_{4}$ ). Tetragonal crystals with a diameter of $0.3 \mathrm{~mm}$ grew within 2 weeks.

Citrate-bound and citrate-free crystals were cryoprotected by transferring them into their respective reservoir solutions supplemented with $30 \%$ glycerol prior to freezing them in liquid nitrogen. Three data sets (peak, inflection and low-energy remote) were collected for SeMet-derivatized citrate-bound CitAP, and one native data set was collected for citrate-free CitAP at DESY Hamburg (BW6; MarCCD detector). All data were processed using HKL2000, ${ }^{26}$ and the space group and data statistics were determined by XPREP (Bruker AXS, Madison, WI). Stat- istics for data collection and processing are summarized in Table 1.

\section{Structure determination and refinement}

Se-Met crystals grown in the presence of citrate crystallized in space group $P 22_{1} 2_{1} 2_{1}$, with two molecules per asymmetric unit. The structure was solved by MAD: normalized difference structure factors were calculated using SHELXC, the substructure was solved using SHELXD ${ }^{27}$ and phase extension and density modification were carried out using SHELXE. ${ }^{28}$ The initial map was excellent, with a mean phase error of $18.1^{\circ}$ and a map correlation coefficient of $93.8 \%$ (Figs. 1e and f). Fifty cycles of automatic model building alternating with structure refinement by ARP/ WARP $^{29}$ resulted in modeling of $94 \%$ of the residues. Refinement was performed by positional and $B$-factor refinement in REFMAC $5^{30}$ alternating with manual model building in Coot, ${ }^{31}$ followed by ARP solvent building. The occupancies of selenium atoms were refined using SHELXL ${ }^{32}$ (Table 1). Crystals grown without adding citrate crystallized in space group I4, with two molecules in the asymmetric unit. Se-Met crystals had a very high mosaicity of 2.2, which made the solution of the structure by MAD or single-wavelength anomalous dispersion difficult. Therefore, the structure was solved by molecular replacement using the program PHASER. ${ }^{33}$ Our structure of the citrate-bound CitAP, without the minor loop (99104) and major loop (68-90) residues, was used as search model. The structure solved with a Z-score of 34 and a loglikelihood gain of 1237.62. Subsequent rigid body refinement with REFMAC5 resulted in high $R$ (42.3) and $R_{\text {free }}$ (43.8) values. Analysis of the data using the Yeates-Fam twinning servert suggested a twin fraction of about 0.2 with the twin law, 010100 00-1. After an initial rigid body refinement in REFMAC $5,{ }^{30}$ more residues could be traced using a bias-minimized map generated by prime and switch phasing in RESOLVE. ${ }^{34}$ A composite omit map calculated using simulated annealing in Crystallography \& NMR System $(\mathrm{CNS})^{35}$ was used for building all minor loop residues and some of the major loop residues. The model was further refined using the twin refinement protocol in CNS by simulated annealing using torsion angle dynamics and energy minimization. As the twin fraction cannot be refined in $\mathrm{CNS}$, the refinement was continued using the twin refinement protocol in SHELXL with an initial twin fraction of 0.2 alternating with model building in Coot (Table 1). Finally, the structure was refined with a beta-test version of the program PHENIX.REFINE, ${ }^{36}$ which can also now handle such twins and had the advantage over SHELXL that the side chain torsion angles could be restrained. Inspection of the Ramachandran backbone dihedral angles in citrate-free CitAP structure revealed a generously allowed backbone conformation for the minor loop residue Ser101 that is involved in citrate binding. This finding is in line with the more frequent occurrence of disallowed residues at ligand-binding sites. ${ }^{37}$ Therefore, this generously allowed residue was not removed from the model of citrate-free CitAP.

\section{NMR spectroscopy}

About $1 \mathrm{mM}{ }^{15} \mathrm{~N},{ }^{13} \mathrm{C}$-labeled CitAP was used for NMR measurements. The protein sample was at $\mathrm{pH}$ 6.0, in $50 \mathrm{mM}$ sodium phosphate buffer. All experiments were 
recorded on Bruker Avance 900- and 600-MHz spectrometers equipped with cryoprobes. The temperature was set to $310 \mathrm{~K}$. For backbone assignments of citrate-free CitAP, the following experiments were performed: threedimensional HNCO, three-dimensional HNCA, threedimensional HNCACB, three-dimensional ${ }^{13} \mathrm{C}$ and ${ }^{15} \mathrm{~N}$ NOE spectroscopy-HSQC (mixing time, $120 \mathrm{~ms}$ ) and three-dimensional CBCACONH. ${ }^{38}{ }^{1} \mathrm{H}-{ }^{15} \mathrm{~N}$ NOE experiments were recorded with 5-s recycling delay. ${ }^{39}$ A 10 -fold excess of sodium citrate was added to the protein to obtain citrate-bound CitAP. For backbone assignments of citrate-bound CitAP, the above experiments for citratefree CitAP were repeated. Data were processed using NMRPipe $^{40}$ and analyzed using SPARKY 3 (T.D. Goddard and D.G. Kneller, SPARKY 3, University of California, San Francisco, San Francisco, CA). Normalized chemical shift differences for amide ${ }^{1} \mathrm{H}$ and ${ }^{15} \mathrm{~N}$ chemical shifts were calculated according to the following equation: $\Delta_{\mathrm{av}}=\left[\left(\Delta \delta_{\mathrm{HN}}^{2}+\Delta \delta_{\mathrm{N}}^{2} / 25\right) / 2\right]^{1 / 2}$.

Citrate-free and citrate-bound CitAP samples were aligned separately in filamentous bacterial phages (Pf1). ${ }^{41}$ The concentration of Pf1 phage in the citrate-free CitAP sample was $10 \mathrm{mg} / \mathrm{ml}$, while that in the citrate-bound CitAP was $12 \mathrm{mg} / \mathrm{ml} .{ }^{1} \mathrm{D}_{\mathrm{NH}}$ couplings for the citrate-bound CitAP sample were measured using an in-phase/antiphase HSQC experiment. ${ }^{42}$ The ${ }^{1} \mathrm{D}_{\mathrm{NH}}$ RDCs were used for comparison of RDCs back-calculated from X-ray structures using the singular value decomposition method as implemented in the program PALES. ${ }^{43}$ Inclusion of the ${ }^{1} \mathrm{D}_{\mathrm{NCO}}$ RDCs did not significantly change the correlation coefficients between experimental and back-calculated RDCs.

\section{Protein Data Bank accession codes}

Coordinates and structure factors have been deposited in the Protein Data Bank with accession codes 2J80 and 2V9A.

\section{Acknowledgements}

This work was supported by Max-PlanckGesellschaft. M.Z. received a Deutsche Forschungsgemeinschaft Heisenberg scholarship (ZW 71/2-1 and 3-1). G.M.S. and C.G. thank the Fonds der Chemischen Industrie for support. This work was also supported by the Deutsche Forschungsgemeinschaft under (GR 1211/13-2). We thank Christina Mack and Karin Giller for their expert technical help. We are also grateful to Gleb Bourenkov and Galina Kachalova for their assistance with $\mathrm{X}$-ray data collection.

\section{Supplementary Data}

Supplementary data associated with this article can be found, in the online version, at doi:10.1016/ j.jmb.2008.01.024

\section{References}

1. West, A. H. \& Stock, A. M. (2001). Histidine kinases and response regulator proteins in two-component signaling systems. Trends Biochem. Sci. 26, 369-376.
2. Bilwes, A. M., Alex, L. A., Crane, B. R. \& Simon, M. I. (1999). Structure of CheA, a signal-transducing histidine kinase. Cell, 96, 131-141.

3. Stock, A. M., Robinson, V. L. \& Goudreau, P. N. (2000). Two-component signal transduction. Annu. Rev. Biochem. 69, 183-215.

4. Miyatake, H., Mukai, M., Park, S. Y., Adachi, S., Tamura, K., Nakamura, H. et al. (2000). Sensory mechanism of oxygen sensor FixL from Rhizobium meliloti: crystallographic, mutagenesis and resonance Raman spectroscopic studies. J. Mol. Biol. 301, 415-431.

5. Rajagopal, S. \& Moffat, K. (2003). Crystal structure of a photoactive yellow protein from a sensor histidine kinase: conformational variability and signal transduction. Proc. Natl Acad. Sci. USA, 100, 1649-1654.

6. Reinelt, S., Hofmann, E., Gerharz, T., Bott, M. \& Madden, D. R. (2003). The structure of the periplasmic ligand-binding domain of the sensor kinase CitA reveals the first extracellular PAS domain. J. Biol. Chem. 278, 39189-39196.

7. Pappalardo, L., Janausch, I. G., Vijayan, V., Zientz, E., Junker, J., Peti, W. et al. (2003). The NMR structure of the sensory domain of the membranous two-component fumarate sensor (histidine protein kinase) DcuS of Escherichia coli. J. Biol. Chem. 278, 39185-39188.

8. Bott, M. (1997). Anaerobic citrate metabolism and its regulation in enterobacteria. Arch. Microbiol. 167, 78-88.

9. Bott, M., Meyer, M. \& Dimroth, P. (1995). Regulation of anaerobic citrate metabolism in Klebsiella pneumoniae. Mol. Microbiol. 18, 533-546.

10. Kaspar, S., Perozzo, R., Reinelt, S., Meyer, M., Pfister, K., Scapozza, L. \& Bott, M. (1999). The periplasmic domain of the histidine autokinase CitA functions as a highly specific citrate receptor. Mol. Microbiol. 33, 858-872.

11. Gerharz, T., Reinelt, S., Kaspar, S., Scapozza, L. \& Bott, M. (2003). Identification of basic amino acid residues important for citrate binding by the periplasmic receptor domain of the sensor kinase CitA. Biochemistry, 42, 5917-5924.

12. Cho, U. S., Bader, M. W., Amaya, M. F., Daley, M. E., Klevit, R. E., Miller, S. I. \& Xu, W. (2006). Metal bridges between the PhoQ sensor domain and the membrane regulate transmembrane signaling. J. Mol. Biol. 356, 1193-1206.

13. Neiditch, M. B., Federle, M. J., Pompeani, A. J., Kelly, R. C., Swem, D. L., Jeffrey, P. D. et al. (2006). Ligandinduced asymmetry in histidine sensor kinase complex regulates quorum sensing. Cell, 126, 1095-1108.

14. Neiditch, M. B., Federle, M. J., Miller, S. T., Bassler, B. L. \& Hughson, F. M. (2005). Regulation of LuxPQ receptor activity by the quorum-sensing signal autoinducer-2. Mol. Cell, 18, 507-518.

15. Michalopoulos, I., Torrance, G. M., Gilbert, D. R. \& Westhead, D. R. (2004). TOPS: an enhanced database of protein structural topology. Nucleic Acids Res. 32, D251-D254.

16. Branden, C. \& Tooze, J. (1999). Introduction to Protein Structure. Garland Publishing, Inc., New York.

17. Tjandra, N. \& Bax, A. (1997). Direct measurement of distances and angles in biomolecules by NMR in a dilute liquid crystalline medium. Science, 278, 1111-1114.

18. Losonczi, J. A. \& Prestegard, J. H. (1998). Improved dilute bicelle solutions for high-resolution NMR of biological macromolecules. J. Biomol. NMR, 12, 447-451.

19. Pos, K. M. \& Dimroth, P. (1996). Functional properties of the purified $\mathrm{Na}(+)$-dependent citrate carrier of 
Klebsiella pneumoniae: evidence for asymmetric orientation of the carrier protein in proteoliposomes. Biochemistry, 35, 1018-1026.

20. Dimroth, P., Jockel, P. \& Schmid, M. (2001). Coupling mechanism of the oxaloacetate decarboxylase $\mathrm{Na}(+)$ pump. Biochim. Biophys. Acta, 1505, 1-14.

21. Muller, P., Kopke, S. \& Sheldrick, G. M. (2003). Is the bond-valence method able to identify metal atoms in protein structures? Acta Crystallogr., Sect. D: Biol. Crystallogr. 59, 32-37.

22. Hulko, M., Berndt, F., Gruber, M., Linder, J. U., Truffault, V., Schultz, A. et al. (2006). The HAMP domain structure implies helix rotation in transmembrane signaling. Cell, 126, 929-940.

23. Matthews, E. E., Zoonens, M. \& Engelman, D. M. (2006). Dynamic helix interactions in transmembrane signaling. Cell, 127, 447-450.

24. Stoddard, B. L., Biemann, H. P. \& Koshland, D. E., Jr (1992). Receptors and transmembrane signaling. Cold Spring Harbor Symp. Quant. Biol. 57, 1-15.

25. Ottemann, K. M., Xiao, W., Shin, Y. K. \& Koshland, D. E., Jr (1999). A piston model for transmembrane signaling of the aspartate receptor. Science, 285, 1751-1754.

26. Otwinowski, Z. \& Minor, W. (1997). Macromolecular crystallography. Methods Enzymol. 276, 307-326.

27. Schneider, T. R. \& Sheldrick, G. M. (2002). Substructure solution with SHELXD. Acta Crystallogr., Sect. D: Biol. Crystallogr. 58, 1772-1779.

28. Sheldrick, G. M. (2002). Macromolecular phasing with SHELXE. Z. Kristallogr. 217, 644-650.

29. Perrakis, A., Morris, R. \& Lamzin, V. S. (1999). Automated protein model building combined with iterative structure refinement. Nat. Struct. Biol. 6, 458-463.

30. Murshudov, G. N., Vagin, A. A. \& Dodson, E. J. (1997). Refinement of macromolecular structures by the maximum-likelihood method. Acta Crystallogr., Sect. D: Biol. Crystallogr. 53, 240-255.

31. Emsley, P. \& Cowtan, K. (2004). Coot: model-building tools for molecular graphics. Acta Crystallogr., Sect. D: Biol. Crystallogr. 60, 2126-2132.

32. Sheldrick, G. M. \& Schneider, T. R. (1997). SHELXL: highresolution refinement. Methods Enzymol. 277, 319-343.

33. McCoy, A. J., Grosse-Kunstleve, R. W., Storoni, L. C. \& Read, R. J. (2005). Likelihood-enhanced fast transla- tion functions. Acta Crystallogr., Sect. D: Biol. Crystal$\log r$. 61, 458-464.

34. Terwilliger, T. C. (2004). Using prime-and-switch phasing to reduce model bias in molecular replacement. Acta Crystallogr., Sect. D: Biol. Crystallogr. 60, 2144-2149.

35. Brunger, A. T., Adams, P. D., Clore, G. M., DeLano, W. L., Gros, P., Grosse-Kunstleve, R. W. et al. (1998). Crystallography \& NMR System: a new software suite for macromolecular structure determination. Acta Crystallogr., Sect. D: Biol. Crystallogr. 54, 905-921.

36. Adams, P. D., Grosse-Kunstleve, R. W., Hung, L. W., Ioerger, T. R., McCoy, A. J., Moriarty, N. W. et al. (2002). PHENIX: building new software for automated crystallographic structure determination. Acta Crystallogr., Sect. D: Biol. Crystallogr. 58, 1948-1954.

37. Gunasekaran, K. \& Nussinov, R. (2007). How different are structurally flexible and rigid binding sites? Sequence and structural features discriminating proteins that do and do not undergo conformational change upon ligand binding. J. Mol. Biol. 365, 257-273.

38. Bax, A. \& Grzesiek, S. (1993). Methodological advances in protein NMR. Acc. Chem. Res. 26, 131-138.

39. Farrow, N. A., Muhandiram, R., Singer, A. U., Pascal, S. M., Kay, C. M., Gish, G. et al. (1994). Backbone dynamics of a free and a phosphopeptide-complexed Src homology-2 domain studied by N-15 NMR relaxation. Biochemistry, 33, 5984-6003.

40. Delaglio, F., Grzesiek, S., Vuister, G. W., Zhu, G., Pfeifer, J. \& Bax, A. (1995). NMRPipe-a multidimensional spectral processing system based on Unix pipes. J. Biomol. NMR, 6, 277-293.

41. Hansen, M. R., Mueller, L. \& Pardi, A. (1998). Tunable alignment of macromolecules by filamentous phage yields dipolar coupling interactions. Nat. Struct. Biol. 5, 1065-1074.

42. Ottiger, M., Delaglio, F. \& Bax, A. (1998). Measurement of $\mathrm{J}$ and dipolar couplings from simplified two-dimensional NMR spectra. J. Magn. Reson. 131, 373-378.

43. Zweckstetter, M. \& Bax, A. (2000). Prediction of sterically induced alignment in a dilute liquid crystalline phase: aid to protein structure determination by NMR. J. Am. Chem. Soc. 122, 3791-3792. 